\title{
Heath State Utility Values for Cost-Effectiveness Models
}

\author{
Jonathan Karnon ${ }^{1}$
}

Published online: 19 October 2017

(c) Springer International Publishing AG 2017

Most contemporary clinical trials of new technologies collect health-related quality of life (HRQoL) data that can be used to generate utility values. Such data can be used to estimate differences in quality-adjusted life-year (QALY) gains in economic evaluations alongside clinical trials. However, such evaluations rarely represent all important differences in costs and outcomes, and cost-effectiveness models are commonly used to extrapolate beyond a trial's time horizon to estimate longer-term costs and QALY gains [1].

HRQoL data collected in clinical trials are potentially important data sources to inform heath state utility values (HSUVs) that are key inputs to most cost-effectiveness models. In the ideal, the utility data collected in a clinical trial provide robust, sensitive preference-based estimates of all the HSUVs required to populate a cost-effectiveness model. Key issues that complicate the estimation of HSUVs using trial data include the sensitivity of selected preference-based measures (PBMs) to meaningful differences in HRQoL between health states, the small samples of utility data for some of the health states represented in cost-effectiveness models and the timing of the collection of utility data in clinical trials. As a result, separate utility studies may be required to more appropriately inform the HSUVs required to populate cost-effectiveness models.

The supplement to this issue of the journal provides a summary and discussion of the wide-ranging methodological and applied issues around the estimation of

Jonathan Karnon

jonathan.karnon@adelaide.edu.au

1 School of Public Health, University of Adelaide, Adelaide, Australia
HSUVs. The authors refer to existing detailed guidance on specific issues, such as methods for mapping utility values to non-preference-based outcome measures, but rightly note there is "no common source that covers the state of the art recommendations across the full spectrum of considerations ... and there are still areas where recommendations for good practice are absent" [2].

Rowen et al. [3] provide an overview of international regulations and recommendations for the collection, analysis and use of utility data. Their paper provides a starting point at which it is useful to differentiate between issues for which best practice can be defined and issues for which alternative regulatory bodies may have legitimately different preferences, such as the use of public or patient preference values. There are arguments in favour of using public and patient values and so a general preference for either is legitimate, whereas the assessment of the sensitivity of alternative PBMs to capturing important differences in HRQoL may be subject to empirical investigation and the development of guidance to support consistency in application.

Concerns around the sensitivity of alternative PBMs may refer to differences in utility between intervention and control patients within the same health state or between alternative health states. Brazier et al. [4] note that the PBM used in an effectiveness trial should be carefully selected, but when is the use of a condition-specific PBM justified (given a preference by reimbursement bodies for generic PBMs)? When is a post hoc claim to disregard insensitive utility data acceptable? When is the available sample of utility data from a clinical trial to inform an HSUV too small?

The collection of utility data during a clinical trial is generally undertaken at fixed timepoints beyond baseline. 
Such data may capture general trends in utility but may not reflect the direct effects of temporary events such as angina events or asthma attacks. Often analysts seek external utility data to represent the utility effects of temporary events, which can be important drivers of estimated QALY gains. What is the most appropriate approach to representing the effects of temporary events: vignette-based general population studies, direct elicitation based on patients' recall of previous events or efforts to capture the utility effects at the time of the event?

The utility effects of treatment-related adverse events can be difficult to handle because of the often wide range of forms and severities experienced [5,6]. Such events are also linked to treatment discontinuation, which may improve utility in the short term. For new therapies and services, clinical trials may be the only source of utility data to describe the effects of treatment-related adverse events and treatment discontinuations. Within each treatment group, the estimation of average utility values for participants at regular intervals may provide relevant data to estimate these utility effects without the need to represent separate treatment-related adverse event states. However, with less than complete utility data, additional analyses that categorise patients by their adverse-event or treatment-continuation status may be required to account for non-random non-responses to utility questions.

A case could be made that trial-based utility data should be used to inform HSUVs prior to the experience of an outcome event represented in a cost-effectiveness model, with other HSUVs being informed by supplementary data collection within a clinical trial or from external data sources. Supplementary data collection within a clinical trial might focus on more targeted and frequent collection of utility data from participants who experience outcome events, to maximise the validity of trial-based estimates of the utility associated with related health states.

Most commonly, analysts will review the existing literature for utility studies that have reported relevant HSUVs. Ara et al. [7] discuss the process of reviewing and synthesising HSUVs from the existing literature, outlining processes that should be followed to select the most appropriate values to be used. In my experience, such a systematic approach to the selection of HSUVs is rarely applied. There is a growing interest in meta-analyses of HSUVs [8], which can reduce the burden on cost-effectiveness modellers and provide a common source of values for economic evaluations across a clinical area. Ara et al. [7] note that "methodological research into the appropriateness of alternative techniques for meta-analysis is in its infancy," but such studies have great potential to improve the quality and consistency of the estimation of HSUVs.

Other data sources for HSUVs with potential for expansion and improved analysis are clinical registries and patient-reported outcome measures (PROMs) programmes such as the NHS England programme, which has been running since 2009. Registries do not commonly collect preference-based HRQoL data. A recent analysis of five Australian registries found only the Victorian Prostate Cancer Registry collected PROMs data, at 12 and 24 months post-diagnosis [9]. The NHS England PROMs programme collects relevant utility data but only to a maximum of 6 months post-discharge. The continuing collection of a minimum dataset describing patients' clinical status and a PROM in clinical registries as well as specific PROMs programmes would provide valuable information to inform long-term outcomes and HSUVs.

In the absence of valid utility data collected within a clinical trial or from existing utility studies, a prospective utility study may be required. To comply with the preferences of decision makers for utilities derived from patientcompleted multi-attribute utility indices, such studies should seek representative populations of patients who are in the health states represented in a cost-effectiveness model. This is difficult, but clinical registries may provide the most appropriate source for the identification of a representative sample.

A final issue concerns the common call for the use of sensitivity analyses to explore the impact of different approaches to the estimation and analysis of HSUVs. The identification of issues to which cost-effectiveness results are not sensitive may provide some assurance to the decision maker, but-in practice-sensitivity to alternative approaches to estimating HSUVs alone is rarely sufficient to delay a funding decision. This situation highlights the importance of a thorough understanding of the validity of alternative approaches to the estimation of HSUVs. The issues highlighted in this editorial and the more detailed assessments in the supplement to the journal illustrate the wide range of factors that can arise when estimating HSUVs.

Current guidance provided by reimbursement bodies make little reference to more detailed HSUV-related methodological issues. Further consideration of these issues will support improvements in the collection of preference-based HRQoL data in clinical studies and better planning and use of supplementary utility studies, where required. In turn, such developments will improve consistency and transparency in decision making.

\section{Compliance with Ethical Standards}

Funding This article is published in a special edition journal supplement wholly funded by Takeda Pharmaceutical International AG, Zurich, Switzerland. The author has not received any payment from Takeda.

Conflicts of interest Jonathan Karnon has no conflicts of interest. 


\section{References}

1. Karnon J, Brennan A, Akehurst R. Decision modeling to inform decision making: seeing the wood for the trees. Med Decis Mak. 2010;30(3):E20-2.

2. Ara R, Peasgood T, Brazier J, Mukuria C, Chevrou-Severac H, Rowen D, et al. Sourcing and using appropriate health state utility values in economic models in health care. Pharmacoeconomics. 2017. doi:10.1007/s40273-017-0543-z.

3. Rowen D, Azzabi Zouraq I, Chevrou-Severac H, van Hout B. International regulations and recommendations for utility data for health technology assessment. Pharmacoeconomics. 2017. doi:10. 1007/s40273-017-0544-y.

4. Brazier J, Ara R, Donna Rowen D, Chevrou-Severac H. A review of generic preference-based measures for use in cost-effectiveness models. Pharmacoeconomics. 2017. doi:10.1007/s40273-0170545-x.
5. Ara R, Brazier J, Azzabi Zouraq I. The use of health state utility values in decision models. Pharmacoeconomics. 2017. doi:10. 1007/s40273-017-0550-0.

6. Ara R, Brazier J. Estimating HSUVs for comorbidities. Pharmacoeconomics. 2017. doi:10.1007/s40273-017-0551-z.

7. Ara R, Brazier J, Peasgood T, Suzy Paisley S. The identification, review and synthesis of HSUVs from the literature. Pharmacoeconomics. 2017. doi:10.1007/s40273-017-0547-8.

8. Peasgood T, Brazier J. Is meta-analysis for utility values appropriate given the potential impact different elicitation methods have on values? Pharmacoeconomics. 2015;33(11):1101-5.

9. The Australian Commission on Safety and Quality in Health Care. Economic evaluation of clinical quality registries: Final report. Sydney: ACSQHC; 2016. 